ELECTRONIC RESEARCH ANNOUNCEMENTS

OF THE AMERICAN MATHEMATICAL SOCIETY

Volume 4, Pages 43-47 (June 11, 1998)

S $1079-6762(98) 00045-6$

\title{
ON CHEREDNIK-MACDONALD-MEHTA IDENTITIES
}

\author{
PAVEL ETINGOF AND ALEXANDER KIRILLOV, JR.
}

(Communicated by David Kazhdan)

\begin{abstract}
In this note we give a proof of Cherednik's generalization of Macdonald-Mehta identities for the root system $A_{n-1}$, using representation theory of quantum groups. These identities give an explicit formula for the integral of a product of Macdonald polynomials with respect to a "difference analogue of the Gaussian measure". They were suggested by Cherednik, who also gave a proof based on representation theory of affine Hecke algberas; our proof gives a nice interpretation for these identities in terms of representations of quantum groups and seems to be simpler than that of Cherednik.
\end{abstract}

\section{INTRODUCTION}

In this note we give a proof of Cherednik's generalization of Macdonald-Mehta identities for the root system $A_{n-1}$, using representation theory of quantum groups. These identities, suggested and proved in [Ch2], give an explicit formula for the integral of a product of Macdonald polynomials with respect to a "difference analogue of the Gaussian measure". They can be written for any reduced root system, or, equivalently, for any semisimple complex Lie algebra g. Assuming for simplicity that $\mathfrak{g}$ is simple and simply laced, these identities are given by the following formula:

$$
\begin{aligned}
\frac{1}{|W|} \int \delta_{k} \overline{\delta_{k}} P_{\lambda} \overline{P_{\mu}} \gamma d x= & q^{\lambda^{2}+(\mu, \mu+2 k \rho)} P_{\mu}\left(q^{-2(\lambda+k \rho)}\right) \\
& \times q^{-2 k(k-1)\left|R_{+}\right|} \prod_{\alpha \in R_{+}} \prod_{i=0}^{k-1}\left(1-q^{2(\alpha, \lambda+k \rho)+2 i}\right)
\end{aligned}
$$

where $\lambda, \mu$ are dominant integral weights, $k$ is a positive integer, $P_{\lambda}$ are Macdonald polynomials associated with the corresponding root system, with parameters $q^{2}, t=$ $q^{2 k}$ (see [M1], [M2] or a review in [Kir2]), $\delta_{k}$ is the $q$-analogue of $k$ th power of the Weyl denominator $\delta=\delta_{1}$ :

$$
\delta_{k}=\prod_{\alpha \in R^{+}} \prod_{i=0}^{k-1}\left(e^{\alpha / 2}-q^{-2 i} e^{-\alpha / 2}\right),
$$

Received by the editors April 14, 1998.

1991 Mathematics Subject Classification. Primary 05E35.

Key words and phrases. Macdonald polynomials. 
and $\gamma$ is the Gaussian, which we define by

$$
\gamma=\sum_{\lambda \in P} e^{\lambda} q^{\lambda^{2}}
$$

where $P$ is the weight lattice. We consider $\gamma$ as a formal series in $q$ with coefficients from the group algebra of the weight lattice. In a more standard terminology $\gamma$ is called the theta-function of the lattice $P$. All other notations, which are more or less standard, will be explained below.

These identities were formulated in the form we use in a paper of Cherednik [Ch2], who also proved them using double affine Hecke algebras (note: our notations are somewhat different from Cherednik's ones). We refer the reader to [Ch2] for the discussion of the history of these identities and their role in difference harmonic analysis.

The proof uses the following identity for the Gaussian, due to Kostant [Kos]:

$$
\gamma=\left(\prod_{\alpha \in R_{+}}\left(1-q^{2(\alpha, \rho)}\right)\right) \sum_{\nu \in P_{+}} q^{(\nu, \nu+2 \rho)}\left(\operatorname{dim}_{q} L_{\nu}\right) \chi_{\nu} .
$$

Here $\chi_{\nu}$ is the character of the irreducible finite-dimensional module $L_{\nu}$ over $\mathfrak{g}$, and $\operatorname{dim}_{q} L_{\nu}:=\chi_{\nu}\left(q^{2 \rho}\right)$ is the quantum dimension of $L_{\nu}$.

Notations. We use the same notations as in [EK1], [EK2] with the following exceptions: we replace $q$ by $q^{-1}$ (note that this does not change Macdonald's polynomials) and we use the notation $\varphi_{\lambda}$ for "generalized characters" (see below), reserving the notation $\chi_{\lambda}$ for usual (Weyl) characters. In particular, we define $\overline{e^{\lambda}}=e^{-\lambda}, \bar{q}=q$, and for $f \in \mathbb{C}_{q}[P]$, we define $f\left(q^{\lambda}\right), \lambda \in P$ by $e^{\mu}\left(q^{\lambda}\right)=q^{(\mu, \lambda)}$. For brevity, we also write $\lambda^{2}$ for $(\lambda, \lambda)$. Finally, we denote by $\int d x: \mathbb{C}_{q}[P] \rightarrow \mathbb{C}_{q}$ the functional of taking the constant term: $\int e^{\lambda} d x=\delta_{\lambda, 0}$.

\section{THE PROOF}

In this section, we give a proof of the Cherednik-Macdonald-Mehta identities (1) for $\mathfrak{g}=\mathfrak{s l}_{n}$. The proof is based on the realization of Macdonald's polynomials as "vector-valued characters" for the quantum group $U_{q} \mathfrak{s l}_{n}$, which was given in [EK1]. For the sake of completeness, we briefly outline these results here, referring the reader to the original paper for a detailed exposition.

Let us fix $k \in \mathbb{Z}_{+}$and denote by $U$ the finite-dimensional representation of $U_{q} \mathfrak{s l}_{n}$ with highest weight $n(k-1) \omega_{1}$, where $\omega_{1}$ is the first fundamental weight. We identify the zero weight subspace $U[0]$, which is one-dimensional, with $\mathbb{C}_{q}$.

For $\lambda \in P_{+}$, we denote by $\Phi_{\lambda}$ the unique intertwiner

$$
\Phi_{\lambda}: L_{\lambda+(k-1) \rho} \rightarrow L_{\lambda+(k-1) \rho} \otimes U
$$

and define the generalized character $\varphi_{\lambda} \in \mathbb{C}_{q}[P] \otimes U[0] \simeq \mathbb{C}_{q}[P]$ by $\varphi_{\lambda}\left(q^{x}\right)=$ $\operatorname{Tr}_{L_{\lambda+(k-1) \rho}}\left(\Phi_{\lambda} q^{x}\right)$.

We can now summarize the results of [EK1] as follows:

$$
\begin{gathered}
\varphi_{0}=\prod_{\alpha \in R_{+}} \prod_{i=1}^{k-1}\left(e^{\alpha / 2}-q^{-2 i} e^{-\alpha / 2}\right)=\delta_{k} / \delta, \\
\varphi_{\lambda} / \varphi_{0}=P_{\lambda},
\end{gathered}
$$

where $P_{\lambda}$ is the Macdonald polynomial with parameters $q^{2}, t=q^{2 k}$. 
We can also rewrite Macdonald's inner product in terms of the generalized characters as follows. Recall that Macdonald's inner product on $\mathbb{C}_{q}[P]$ is defined by

$$
\langle f, g\rangle_{k}=\frac{1}{|W|} \int \delta_{k} \overline{\delta_{k}} f \bar{g} d x
$$

(this differs by a certain power of $q$ from the original definition of Macdonald). Obviously, one has

$$
\left\langle P_{\lambda}, P_{\mu}\right\rangle_{k}=\left\langle\varphi_{\lambda}, \varphi_{\mu}\right\rangle_{1}
$$

In order to rewrite this in terms of representation theory, let $\omega$ be the Cartan involution in $U_{q} \mathfrak{s l}_{n}$ (see [EK1]). For a $U_{q} \mathfrak{s l}_{n}$-module $V$, we denote by $V^{\omega}$ the same vector space but with the action of $U_{q} \mathfrak{s l}_{n}$ twisted by $\omega$. Note that for finitedimensional $V$, we have $V^{\omega} \simeq V^{*}$ (not canonically). Similarly, for an intertwiner $\Phi: L \rightarrow L \otimes U$ we denote by $\Phi^{\omega}$ the corresponding intertwiner $L^{\omega} \rightarrow U^{\omega} \otimes L^{\omega}$. Finally, for $\Phi_{1}: L_{1} \rightarrow L_{1} \otimes U, \Phi_{2}: L_{2} \rightarrow L_{2} \otimes U$, define $\Phi_{1} \odot \Phi_{2}^{\omega} \in \operatorname{End}\left(L_{1} \otimes L_{2}^{\omega}\right)$ as the composition $L_{1} \otimes L_{2}^{\omega} \rightarrow L_{1} \otimes U \otimes U^{\omega} \otimes L_{2}^{\omega} \rightarrow L_{1} \otimes L_{2}^{\omega}$, where the first arrow is given by $\Phi_{\lambda} \otimes \Phi_{\mu}^{\omega}$, and the second by the invariant pairing $U \otimes U^{\omega} \rightarrow \mathbb{C}_{q}$ (which is unique up to a constant). Then it was shown in [EK1] that

$$
\left(\varphi_{\lambda} \overline{\varphi_{\mu}}\right)\left(q^{x}\right)=\operatorname{Tr}_{V}\left(\left(\Phi_{\lambda} \odot \Phi_{\mu}^{\omega}\right) q^{\Delta(x)}\right)=\sum_{\nu \in P_{+}} \chi_{\nu}\left(q^{x}\right) C_{\lambda \mu}^{\nu}
$$

where $V=L_{\lambda+(k-1) \rho} \otimes L_{\mu+(k-1) \rho}^{\omega}$ and $C_{\lambda \mu}^{\nu}$ is the trace of $\Phi_{\lambda} \odot \Phi_{\mu}$ acting in the multiplicity space $\operatorname{Hom}\left(L_{\nu}, V\right)$. As a corollary, we get the following result:

$$
\frac{1}{|W|} \int \delta \bar{\delta} \varphi_{\lambda} \overline{\varphi_{\mu}}\left(\sum_{\nu \in P^{+}} a_{\nu} \chi_{\nu}\right) d x=\sum_{\nu \in P^{+}} a_{\nu^{*}} C_{\lambda \mu}^{\nu}
$$

where $\nu^{*}=-w_{0}(\nu)$ is the highest weight of the module $\left(L_{\nu}\right)^{*}$ (here $w_{0}$ is the longest element of the Weyl group).

Of course, the coefficients $C_{\lambda \mu}^{\nu}$ are very difficult to calculate. However, the formula above is still useful. For example, it immediately shows that $\left\langle\varphi_{\lambda}, \varphi_{\mu}\right\rangle_{1}=0$ unless $\lambda=\mu$, which was the major part of the proof of the formula $\varphi_{\lambda} / \varphi_{0}=P_{\lambda}$ in [EK1]. It turns out that this formula also allows us to prove the CherednikMacdonald-Mehta identities.

Theorem 1. Let $\varphi_{\lambda}$ be the renormalized Macdonald polynomials for the root system $A_{n-1}$ given by (5), and let $\gamma$ be the Gaussian (3). Then

$$
\begin{aligned}
\frac{1}{|W|} \int \delta \bar{\delta} \varphi_{\lambda} \overline{\varphi_{\mu}} \gamma d x= & q^{(\lambda+k \rho)^{2}} q^{(\mu+k \rho)^{2}} \varphi_{\mu}\left(q^{-2(\lambda+k \rho)}\right) \\
& \times\left(\prod_{\alpha \in R_{+}}\left(1-q^{2(\alpha, \rho)}\right)\right) q^{-2 \rho^{2}}\left\|P_{\lambda}\right\|^{2} \operatorname{dim}_{q} L_{\lambda+(k-1) \rho},
\end{aligned}
$$

where $\left\|P_{\lambda}\right\|^{2}=\left\langle P_{\lambda}, P_{\lambda}\right\rangle_{k}$.

Proof. From (6) and (4), we get

$$
\int \delta \bar{\delta} \varphi_{\lambda} \overline{\varphi_{\mu}} \gamma d x=\left(\prod_{\alpha \in R_{+}}\left(1-q^{2(\alpha, \rho)}\right)\right) \sum_{\nu \in P^{+}} q^{(\nu, \nu+2 \rho)}\left(\operatorname{dim}_{q} L_{\nu}\right) C_{\lambda \mu}^{\nu} .
$$


On the other hand, let $C$ be the Casimir element for $U_{q} \mathfrak{g}$ discussed above. Consider the intertwiner $\left(\Phi_{\lambda} \odot \Phi_{\mu}^{\omega}\right) \Delta(C): V \rightarrow V$, where, as before, $V=L_{\lambda+(k-1) \rho} \otimes$ $L_{\mu+(k-1) \rho}^{\omega}$. Then it follows from $\left.C\right|_{L_{\lambda}}=q^{(\lambda, \lambda+2 \rho)}$ that

$$
\operatorname{Tr}_{V}\left(\left(\Phi_{\lambda} \odot \Phi_{\mu}^{\omega}\right) \Delta(C) \Delta\left(q^{2 \rho}\right)\right)=\sum_{\nu \in P_{+}} C_{\lambda \mu}^{\nu} q^{(\nu, \nu+2 \rho)} \operatorname{dim}_{q} L_{\nu},
$$

which is exactly the sum on the right-hand side of (8). On the other hand, using $\Delta(C)=(C \otimes C)\left(R^{21} R\right)$, we can write

$$
\begin{aligned}
\operatorname{Tr}_{V} & \left(\left(\Phi_{\lambda} \odot \Phi_{\mu}^{\omega}\right) \Delta(C) \Delta\left(q^{2 \rho}\right)\right) \\
& =q^{-2 \rho^{2}} q^{(\lambda+k \rho)^{2}} q^{(\mu+k \rho)^{2}} \operatorname{Tr}_{V}\left(\left(\Phi_{\lambda} \odot \Phi_{\mu}^{\omega}\right)\left(R^{21} R\right) \Delta\left(q^{2 \rho}\right)\right) .
\end{aligned}
$$

This last trace can be calculated, which was done in [EK2, Corollary 4.2], and the answer is given by

$$
\operatorname{Tr}_{V}\left(\left(\Phi_{\lambda} \odot \Phi_{\mu}^{\omega}\right)\left(R^{21} R\right) \Delta\left(q^{2 \rho}\right)\right)=\varphi_{\mu}\left(q^{-2(\lambda+k \rho)}\right)\left\|P_{\lambda}\right\|^{2} \operatorname{dim}_{q} L_{\lambda+(k-1) \rho} .
$$

Combining these results, we get the statement of the theorem.

The norms $\left\|P_{\lambda}\right\|^{2}$ appearing on the right-hand side of (7) are given by Macdonald's inner product identities

$$
\left\|P_{\lambda}\right\|^{2}=\prod_{\alpha \in R_{+}} \prod_{i=1}^{k-1} \frac{1-q^{-2(\alpha, \lambda+k \rho)-2 i}}{1-q^{-2(\alpha, \lambda+k \rho)+2 i}},
$$

which were conjectured in [M1], [M2] and proved for the root system $A_{n-1}$ by Macdonald himself [M3]; see also [EK2] for the proof based on representation theory of $U_{q} \mathfrak{s l}_{n}$, and [Ch1] or a review in [Kir2] for a proof for arbitrary root systems. Using this formula and rewriting the statement of Theorem 1 in terms of Macdonald polynomials $P_{\lambda}$ rather than $\varphi_{\lambda}$, we get the Cherednik-Macdonald-Mehta identities (1).

Remarks. 1. Note that the left-hand side of (7) is symmetric in $\lambda, \mu$. Thus, the same is true for the right-hand side, which is exactly the statement of Macdonald's symmetry identity (compare with the proof in [EK2]).

2. The proof of Cherednik-Macdonald-Mehta identities given above easily generalizes to the case when $q$ is a root of unity (see [Kir1] for the discussion of the appropriate representation-theoretic setup). In this case, we need to replace the set $P_{+}$of all integral dominant weights by an appropriate (finite) Weyl alcove $C$ (see [Kir1]), and the integral $\int \delta \bar{\delta} f d x$ should be replaced by const $\sum_{\lambda \in C} f\left(q^{2(\lambda+\rho)}\right) \operatorname{dim}_{q} L_{\lambda}$. Using the following obvious property of the Gaussian:

$$
\gamma\left(q^{2(\lambda+\rho)}\right)=q^{-(\lambda, \lambda+2 \rho)} \gamma\left(q^{2 \rho}\right)
$$

(which in this case coincides with formula (1.7) in [Kir1]), it is easy to see that in this case the Cherednik-Macdonald-Mehta identities are equivalent to

$$
S^{-1} T^{-1} S=T S T,
$$

where the matrices $S, T$ are defined in [Kir1, Theorem 5.4]. This identity is part of a more general result, namely, that these matrices $S, T$ give a projective representation of the modular group $S L_{2}(\mathbb{Z})$ on the space of generalized characters (see [Kir1, Theorem 1.10] and references therein). 


\section{REFERENCES}

[Ch1] I. Cherednik, Double affine Hecke algebras and Macdonald's conjectures, Annals of Math. 141 (1995), 191-216. MR 96m:33010

[Ch2] _ Difference Macdonald-Mehta conjecture, Internat. Math. Res. Notices 1997, 449467. CMP 97:12

[EK1] P. Etingof and A. Kirillov, Jr., Macdonald's polynomials and representations of quantum groups, Math. Res. Let. 1 (1994), 279-296. MR 96m:17025

[EK2] _ Representation-theoretic proof of the inner product and symmetry identities for Macdonald's polynomials, Compos. Math. 102 (1996), 179-202. MR 97j:17013

[Kas] C. Kassel, Quantum groups, Springer-Verlag, New York, 1995. MR 96e:17041

[Kir1] A. Kirillov, Jr., On an inner product in modular tensor categories, J. Amer. Math. Soc. 9 (1996), 1135-1169. MR 97f: 18007

[Kir2] _ Lectures on affine Hecke algebras and Macdonald's conjectures, Bull. Amer. Math. Soc. 34 (1997), 251-292. CMP 97:13

[Kos] B. Kostant, On Macdonald's $\eta$-function formula, the Laplacian and generalized exponents, Advances in Math. 20 (1976), 179-212. MR 58:5484

[M1] I. G. Macdonald, A new class of symmetric functions, Publ. I.R.M.A. Strasbourg, 372/S-20, Actes 20 Séminaire Lotharingien (1988), 131-171.

[M2] Orthogonal polynomials associated with root systems, preprint (1988).

[M3] _ Symmetric functions and Hall polynomials, 2nd ed., The Clarendon Press, Oxford University Press, New York, 1995. MR 96h:05207

Department of Mathematics, Harvard University, Cambridge, MA 02138

E-mail address: etingof@math.harvard.edu

Department of Mathematics, Mit, Cambridge, MA 02139

E-mail address: kirillov@math.mit.edu 